\title{
Eventos de Vida Constituem um Construto? Evidências da Impossibilidade de Considerar Eventos de Vida um Construto
}

\author{
Carla Woyciekoski ${ }^{1}$ \\ Desenvolvimento de Pessoas do Hospital de Clínicas de Porto Alegre, Porto Alegre, \\ Rio Grande do Sul, Brasil \\ Laboratório de Mensuração do Instituto de Psicologia da Universidade Federal \\ do Rio Grande do Sul, Porto Alegre, Rio Grande do Sul, Brasil \\ Jean Carlos Natividade \\ Laboratório de Mensuração do Instituto de Psicologia da Universidade Federal \\ do Rio Grande do Sul, Porto Alegre, Rio Grande do Sul, Brasil \\ Programa de Pós-Graduação em Psicologia da Universidade Federal do Rio Grande do Sul, \\ Porto Alegre, Rio Grande do Sul, Brasil

\section{Claudio Simon Hutz} \\ Departamento de Psicologia do Desenvolvimento e da Personalidade da Universidade \\ Federal do Rio Grande do Sul, Porto Alegre, Rio Grande do Sul, Brasil
}

\section{Resumo}

Eventos de vida (EV) referem-se a experiências vitais, de ordem física e ou psicológica, que podem representar mudanças significativas ou discretas na vida das pessoas. O presente estudo visou discutir a avaliação de EV, bem como à construção e validação de construto de uma escala para a mensuração da percepção (avaliação subjetiva) de EV. Investigou-se se EV se agrupavam em dimensões, conforme categorias relatadas na literatura. Participaram 619 universitários, de ambos os sexos. Utilizou-se um questionário com perguntas sociodemográficas e uma lista com 53 eventos de vida para que os participantes indicassem quais eventos já ocorreram com eles e quão positivos e negativos foram esses eventos. As análises fatoriais mostraram não ser adequado extrair fatores, demonstrando que EV não se agrupam em dimensões e, portanto, não constituem um construto. Verificou-se a existência de tendências quanto à positividade ou negatividade na avaliação de EV. Os resultados reforçam a necessidade de considerar-se na pesquisa em EV, a avaliação subjetiva dos eventos, além de sua ocorrência.

Palavras-chave: Eventos de vida, instrumentos de medida, validade.

\section{Do Life Events Constitute a Construct? Evidence of the Impossibility to Consider Life Events a Construct}

\footnotetext{
Abstract

Life events (LE) refer to vital experiences, physical or psychological, that may represent discrete or significant changes in people's lives. The present study aimed to discuss the evaluation of LE; create a scale

Endereço para correspondência: Laboratório de Mensuração, Instituto de Psicologia, Universidade Federal do Rio Grande do Sul, Rua Ramiro Barcelos, 2.600, Sala 101, Santa Cecília, Porto Alegre, RS, Brasil 90035-003. E-mail: carwpsi@hotmail.com,jeannatividade@gmail.com e claudio.hutz@terra.com.br
} 
for measuring perceived (subjective evaluation) LE as well as obtain construct validation. Further it was investigated whether LE are gathered in dimensions according to categories described in the literature. The participants were 619 university students of both sexes. Factor analyzes were conducted, but factors were not extracted, demonstrating that LE do not constitute a construct. It was verified the presence of trends regarding the positive and negative evaluation of LE. The results point to the importance of considering the subjective evaluation as well as the occurrence of events in LE evaluation.

Keywords: Life-events, measurement instruments, validity.

\section{Eventos de la Vida Constituyen un Constructo? Pruebas de la Imposibilidad de se Considerar los Eventos de la Vida un Constructo}

\section{Resumen}

Eventos de la vida (EV) se refieren a experiencias vitales, de cuño físico y/o psicológico, que pueden representar cambios significativos o discretos en la vida de las personas. El presente estudio tuve como objetivo discutir la evaluación de EV, así como la construcción y validación de constructo de una escala para medir la percepción (evaluación subjetiva) de EV. Además, se investigó si EV si agrupan en dimensiones como categorías reportados en la literatura. Participaron 619 estudiantes universitarios de ambos sexos. Se utilizó un cuestionario con preguntas demográficas y de una lista con 53 eventos de la vida, en que los participantes tenían que indicar se ya habían vivido cada evento y como evaluaban la positividad y negatividad de los eventos. Los análisis factoriales mostraran no ser adecuado extraer factores, lo que demuestra que los EV no son un constructo. Se encontró que hay tendencias cuanto a la positividad o negatividad de la evaluación de EV. Los resultados refuerzan la necesidad de la evaluación subjetiva de los eventos ser considerada en la investigación de EV, aparte de su ocurrencia.

Palabras clave: Eventos de la vida, instrumentos de medición, validez.

Em sua definição inicial, Eventos de Vida (EV) foram descritos como ocorrências do cotidiano, de ordem física e ou psicológica, que modificam ou ameaçam modificar atividades rotineiras (Brown \& Harris, 1978). Foram também definidos como acontecimentos que representam mudanças discretas na vida pessoal ou social (Paykel, 1994; Rafanelli et al., 2005); ou mudanças importantes como o início de um novo emprego, casar-se, separar-se, sofrer um acidente etc (Margis, Picon, Cosner, \& Silveira, 2003). Esses eventos são geralmente classificados na literatura como positivos (nascimento de um filho e casamento, por exemplo) ou negativos (doença ou morte de alguém próximo, por exemplo; Lüdtke, Roberts, Trautwein, \& Nagy, 2011).

Os EV têm sido investigados a partir de duas perspectivas: a perspectiva do estresse e a do desenvolvimento (Luhmann, Hofmann, Eid, \& Lucas, 2012). A partir da primeira, EV são tratados como podendo causar estresse. Sendo assim, EV são denominados estressantes se causarem mudanças e demandas de reajustamento da rotina normal de uma pessoa (Schimmack \& Diener, 2003). Há, ainda, menção aos eventos de vida crônicos (os que envolvem tensão crônica) e traumáticos graves, os quais acarretam o comprometimento da integridade física do próprio indivíduo ou de outrem (Margis et al., 2003).

$\mathrm{Na}$ perspectiva do desenvolvimento, EV demarcam transições específicas na vida das pessoas (Luhmann et al., 2012). Uma transição implicaria numa descontinuidade na vida de um indivíduo, requerendo novas respostas comportamentais. Sendo assim, Luhmann et al. (2012) definem os EV como transições que demarcam o começo ou final de um status específico. Exemplos de status são estado civil (solteiro, casado, divorciado, separado, viúvo) e situação ocupacional (empregado, desempregado, estudando). 
Ainda, mais recentemente, foi proposta a diferenciação dos EV como dependentes ou independentes (Garcia, 2007). Os primeiros envolvem a participação do sujeito na promoção do evento, tal que seu comportamento provoca situações favoráveis ou desfavoráveis para si mesmo. Os EV independentes são aqueles que independem de sua participação, estão além do controle de um indivíduo, sendo inevitáveis, como por exemplo, a morte de um familiar num acidente (Margis et al., 2003).

Os EV são comumente mensurados por meio de listas padronizadas, escalas e entrevistas estruturadas. As listas deram início aos estudos envolvendo $\mathrm{EV}$ e foram e são amplamente utilizadas na pesquisa científica (Dohrenwend, 2006). A primeira lista de EV foi desenvolvida por Adolf Meyer, que na década de 1940 organizou uma lista de eventos para auxiliá-lo na coleta de informações sobre seus pacientes (Kessler, 1997).

Holmes e Rahe (1967) aprimoraram o método e criaram a escala de Reajustamento Social (Social Readjustment Rating Scale - SRRS), um questionário autoadministrado que listava 43 experiências estressantes, encontradas em pesquisas clínicas que identificavam os EV que mais ocorriam aos pacientes antes de eles buscarem auxílio médico. Os indivíduos assinalavam eventos vivenciados em um passado recente, que abrangia de seis meses a um ano. Nesta escala, cada item recebia um valor fixado por juízes, através de um cuidadoso levantamento com um grande número de pessoas (Garcia, 2007).

Dohrenwend (1985) definiu o estresse como um estímulo ambiental independente do estado emocional ou reação da pessoa a um evento. $\mathrm{O}$ autor desenvolveu a Psychiatric Epidemiology Research Interview (PERI - Dohrenwend, 1998), na qual, para cada EV eram emitidos valores (escores) gerados por juízes, desconsiderando-se o que a pessoa reportava sobre sua resposta ao evento. Avaliadores julgavam como a maioria dos indivíduos se sentiria em situações similares e, assim, EV recebiam um valor geral padrão, pontuado sempre que o evento em questão fosse assinalado pelo respondente.

Similarmente, diversos autores (Paykel, 1994; Rafanelli et al., 2005) defenderam que
EV deveriam ser tratados como fatos externos e verificáveis, e não internos e psicológicos. Ressalta-se que, de acordo com esses modelos de avaliação dos EV, não se considera a percepção dos indivíduos sobre os eventos. Reich e Zautra (1981) criticaram a ausência de quantificação do impacto dos EV para os sujeitos. Assim como já haviam indicado Sarason, Johnson e Siegel (1978), faz-se necessário individualizar os escores relativos aos eventos vividos, uma vez que indivíduos podem perceber e experienciar eventos distintamente.

Sarason et al. (1978) propuseram que o valor dos EV deveria variar em termos de sua desejabilidade, ou seja, dependendo das circunstâncias e da percepção do respondente. Eles desenvolveram a escala denominada Life Experiences Survey (LES), na qual o respondente indicava se o evento foi positivo ou negativo e qual o seu valor em uma escala de -3 (muito negativo) a +3 (muito positivo). Reich e Zautra (1981) resumiram os principais achados dessa linha de pesquisa: os mesmos EV podiam ser positivos ou negativos para distintos sujeitos; ter ocorrido num passado remoto ou num passado recente, e ser vistos como estando sob o controle de quem os vivenciou, ou como algo que escapava ao seu controle.

Há ainda estudos que utilizam questionários ou entrevistas estruturadas (Oliveira, Fonseca, \& Del Porto, 2002; Tessner, Mittal, \& Walker, 2011). Brown e Harris (1978) inauguraram o uso de entrevistas, criando a Life Events and Diffculties Schedule (LEDS). Segundo Dohrenwend (2006) ela foi e ainda consiste na entrevista mais utilizada dentro dessa abordagem. Os autores desenvolveram um método que utilizava uma planilha de EV estressantes, para os quais eram dados diferentes escores atribuídos por juízes. Por meio de entrevistas aprofundadas, identificavam-se os fatos e características que se relacionavam aos eventos, a fim de atribuir escores aos mesmos.

Esse método tem sido criticado por ser pouco econômico, trabalhoso, requerer muito tempo, além de demandar investigadores treinados para a coleta das informações acerca dos EV estressantes. Devido a essas dificuldades, 
observa-se a preferência por checklists e escalas, as quais têm predominado na pesquisa científica sobre EV. Essas, por sua vez, também têm sido alvo de críticas devido à abrangência dos itens e à falta de especificidade e sensibilidade na definição de alguns eventos. Também foram observados problemas de fidedignidade e validade (Dohrenwend, 2006). Ainda, é importante destacar que a maioria dos estudos envolvendo EV não fornecem informações sobre as propriedades psicométricas dos instrumentos utilizados.

Todavia, na literatura, por meio de vários estudos tem-se reportado a importância da aplicabilidade das listas de EV na pesquisa e no diagnóstico clínico médico, psicológico e psiquiátrico. Por exemplo, há indicações da relação entre a ocorrência de EV e saúde, bem-estar subjetivo, depressão e a presença de transtornos psicológicos e psiquiátricos. Contudo, essa relação não está clara, pois alguns autores preconizam uma relação causal de eventos produzindo afecções psíquicas e sintomatologia médica (Garcia, 2007; Jordanova et al., 2007; Margis et al., 2003), enquanto outros ressaltam o papel dos traços de personalidade, bem como de outras variáveis, interferindo nessa relação, e até, produzindo os eventos (Chalise, Saito, Takahashi, \& Kai, 2007; Diener \& Oishi, 2005; Garcia, 2007; Headey \& Wearing, 1989).

Dentre os desafios metodológicos, destacam-se as dificuldades na avaliação dos eventos e o nível de independência desses de sintomas e quadros patológicos (Leskela et al., 2004; Paykel, 2003). A partir da revisão de literatura, dentre os principais problemas relativos à mensuração dos EV, destaca-se que:

1. Em alguns instrumentos, EV são tratados como se fossem construtos, uma vez que são gerados escores de EV positivos ou negativos, mas sem apresentar evidências de validade de conteúdo (por exemplo, a Life Events Inventory, a SRLE, a SLE e versões posteriores). Destaca-se que esses eventos são codificados como positivos, negativos ou estressantes pelo próprio pesquisador, sem levar em conta a avaliação do sujeito (ver Faravelli, Catena, Scarpato, \& Ricca, 2007; Fortes-Burgos, Neri, \& Cupertino, 2009; Kohn et al., 2001; Morse \& Robins, 2005; Spurgeon, Jackson, \& Beach, 2001; Voss, Stegmann, \& Schroder, 2006).

2. Alguns instrumentos que incluem a avaliação do impacto dos EV fornecem valores aos eventos a priori com base no julgamento de juízes, sem considerar o impacto do evento para o sujeito. No caso de entrevistas, o impacto é avaliado pelo entrevistador. Por exemplo, a LEDS, o Schedule of Recent Experience, a SRLE e versões posteriores, a PERI; a Interview for Recent Life Events - Paykel, Rao e Taylor (1984; ver também Farmer et al., 2002; Fountoulakis, Iacovides, Kaprinis, \& Kaprinis, 2006; Oliveira et al., 2002; Sheril et al., 2008; Tessner et al., 2011).

3. Em alguns estudos são criadas categorias de eventos, com base na análise de juízes, sem referências à validade de construto (ver Bifulco et al., 1989; Gomez, Krings, Bangerter, \& Grob, 2009; Kolarik, 2010; Lüdtke et al., 2011; Murray \& Cox, 2005; Paykel, 1997; Savoia \& Bernik, 2004; Tessner et al., 2011; Voss et al., 2006).

4. Listas de eventos estressantes ou adversos contêm alguns eventos que não necessariamente são considerados estressantes por todos. Numa pesquisa conduzida por Brown e Harris (1978), itens como "separação", "divórcio", "mudança de casa" compõem a lista de eventos estressores. Em outro estudo conduzido por Fortes-Burgos et al. (2009), aposentadoria foi considerada um evento estressante. Nesses estudos não há avaliação do impacto dos eventos pelos respondentes. Outro estudo conduzido por Dell'Aglio, Benetti, Deretti, D'Incao e Leon (2005), em que se utilizou uma versão adaptada do Inventário de eventos Estressores na Adolescência, itens como "um dos pais se casar novamente", "um dos pais ter filhos com outros parceiros", "mudar de colégio", "ter provas no colégio" são denominados eventos estressores. Nota-se que na avaliação 
do impacto desses eventos, a maioria dos respondentes considerou esses itens como de baixo impacto. Por exemplo, 63,9\% dos adolescentes que vivenciaram "um dos pais ter filhos com outros parceiros", indicaram que esse evento teve baixo impacto, e $22,2 \%$ avaliou esse item como tendo alto impacto. Dos que vivenciaram ter provas no colégio, $55,3 \%$ consideraram o evento de baixo impacto.

5. A maioria dos instrumentos que consideram a avaliação do sujeito sobre os eventos não avalia a positividade dos eventos. Em geral as escalas oferecem a opção "nada estressante" ou "nada negativo"; "muito estressante" ou "muito negativo" (ver, por exemplo a LES e a SLE - Kolarik, 2010; Sarason et al., 1978).

6. Observa-se que na mensuração dos eventos não tem sido avaliada a positividade e negatividade dos eventos separadamente. Avalia-se a neutralidade, a positividade ou a negatividade (ver Lüdtke et al., 2011). Em outras escalas, o respondente deve optar por positivo, neutro ou negativo (Gomez et al., 2009).

7. São poucos os estudos que, ao correlacionar EV com outras variáveis, consideram os eventos individualmente (ver Gomez et al., 2009; Kolarik, 2010; Lüdtke et al., 2011).

$\mathrm{O}$ avanço do conhecimento acerca de um fenômeno depende da existência de instrumentos de medida válidos e confiáveis. No Brasil, não há nenhuma escala com evidências de validade que acesse a percepção ou avaliação subjetiva de EV, considerando, separadamente, o impacto positivo e negativo dos eventos para o sujeito que os experimentou.

Considerando-se o atual panorama da pesquisa em EV, os objetivos do presente estudo foram: (a) verificar se EV constituem um construto que possa ser mensurado objetivamente, uma vez que alguns estudos tratam eventos como se fossem construtos; (b) Verificar se eventos se agrupam em dimensões, conforme categorias relatadas na literatura e (c) verificar a existência de tendências quanto a positividade ou negatividade na avaliação dos EV.

\section{Método}

\section{Construção da Lista de Eventos de Vida}

Inicialmente foram consultadas as bases do Web of Science, onde se encontraram artigos envolvendo a temática de EV, bem como identificaram-se as escalas de EV disponíveis internacionalmente (publicadas em português, inglês e espanhol). Após reunir todos os itens das 13 escalas obtidas, foi organizada uma planilha com 284 itens. Em seguida, os itens similares foram agrupados, e foram retirados itens que dificilmente seriam endossados por pessoas de nossa amostra; obtendo-se uma lista de 53 itens.

Paralelamente, foi realizado um estudo exploratório com 20 participantes, aos quais solicitou-se que escrevessem uma lista de eventos de vida positivos, e negativos que eles tivessem experienciado ou que pudessem, simplesmente, imaginar. Foi produzida uma lista com os eventos sugeridos por eles, e comparou-se essa lista com a lista dos eventos da literatura. Os participantes do estudo piloto não mencionaram nenhum evento que já não tivesse sido contemplado na lista de 53 itens da literatura.

Ao final, os itens foram descritos na forma de acontecimentos e foram apresentados para três pessoas para avaliar sua compreensibilidade e clareza. Os itens considerados confusos, incompreensíveis, ambíguos ou que apresentavam problemas na sua construção foram reelaborados. A versão final contou com 53 itens. As instruções foram descritas de forma a assegurar o correto preenchimento da lista, e para sua melhor compreensão foi oferecido um exemplo.

\section{Verificação de Evidências de Validade de Construto}

Participantes. Participaram 619 estudantes universitários escolhidos por conveniência, com média de idade de 26,4 anos $(D P=9,40)$, $68 \%$ eram mulheres, todos provenientes de três universidades da região metropolitana de Porto Alegre - RS.

Instrumentos. Utilizou-se um questionário contendo perguntas sociodemográficas e uma 
lista com 53 eventos de vida. São exemplos de eventos: conclusão de curso universitário; demissão; conflitos com o parceiro. Ao lado de cada evento os participantes deveriam indicar se ele ocorreu em suas vidas e julgar quão intenso positivamente e negativamente foi o evento, em duas escalas de cinco pontos, tal que quanto mais próximo do cinco, mais intenso seria o evento.

Procedimentos. A coleta foi realizada de forma coletiva em duas universidades privadas e uma pública da região metropolitana de Porto Alegre. Os questionários foram preenchidos individualmente e aplicados nas salas de aula das instituições. Os pesquisadores colocaram-se a disposição para dirimir dúvidas relacionadas à maneira de responder as questões. A pesquisa obteve aprovação do comitê de ética em pesquisa com seres humanos da Universidade Federal do Rio Grande do Sul.

\section{Resultados}

Os itens de eventos de vida apresentavam uma escala para avaliar a positividade do evento e uma para avaliar a negatividade do evento. A fim de testar se os EV agrupavam-se em dimensões, representando construtos, realizaram-se análises de componentes principais com rotação oblíqua com os escores tanto da avaliação positiva quanto da avaliação negativa dos eventos. Essa análise ainda foi realizada para os valores obtidos com a multiplicação dos escores das duas escalas, com o objetivo de agrupar os eventos pela intensidade, independentemente da avaliação positiva ou negativa. Foram realizadas, portanto, três distintas análises de componentes principais em que se incluíram todos os 53 itens correspondentes a eventos de vida.

O baixo número de eventos comuns aos respondentes impossibilitou a realização das análises de componentes principais incluindo todos os itens do instrumento, tendo-se em vista a impossibilidade de gerar matrizes de correlação. Uma alternativa para tornar viável essa análise seria substituir os escores dos itens faltantes, porém se descartou essa possibilidade devido ao alto número de escores hipotéticos que seriam gerados. Como pode ser visto na Tabela 1, apenas 17 dos 53 eventos da lista foram assinalados por mais de $50 \%$ dos participantes.

Tabela 1

FrequênciadosParticipantesqueAssinalouCadaEventodeVidaeMédiadaAvaliaçãoPositivaeNegativa

\begin{tabular}{lccccccc}
\hline Eventos & Participantes & $\begin{array}{c}\text { Avaliação } \\
\text { Positiva }\end{array}$ & $\begin{array}{c}\text { Avaliação } \\
\text { Negativa }\end{array}$ & \\
\hline & $\%$ & $N$ & $M$ & $D P$ & $M$ & $D P$ & Teste Estatístico \\
\hline 1. Ingresso curso universitário & 98,7 & 611 & 4,71 & 0,59 & 1,29 & 0,66 & $t(603)=81,6 ; p<0,001 ; d=5,44$ \\
11. Início de relação amorosa & 91,3 & 565 & 4,33 & 0,96 & 1,89 & 1,11 & $t(556)=32,0 ; p<0,001 ; d=2,33$ \\
31. Viagem & 82,4 & 510 & 4,70 & 0,69 & 1,45 & 0,84 & $t(500)=53,9 ; p<0,001 ; d=4,28$ \\
12. Término de relação amorosa* & 76,3 & 472 & 2,99 & 1,43 & 3,15 & 1,41 & $t(468)=-1,36 ; p=0,18 ; d=-0,09$ \\
29. Mudança de residência & 75,1 & 465 & 4,13 & 1,12 & 2,01 & 1,15 & $t(459)=21,9 ; p<0,001 ; d=1,85$ \\
39. Falecimento de familiar & 74,2 & 459 & 1,36 & 0,76 & 4,42 & 1,02 & $t(455)=-42,3 ; p<0,001 ; d=-3,46$ \\
14. Conflitos com parceiro & 69,5 & 430 & 2,37 & 1,35 & 3,63 & 1,17 & $t(426)=-11,6 ; p<0,001 ; d=-0,99$ \\
28. Melhoria no relacionamento familiar & 66,9 & 414 & 4,66 & 0,72 & 1,30 & 0,74 & $t(407)=53,6 ; p<0,001 ; d=4,56$ \\
32. Familiar doente & 65,8 & 407 & 1,40 & 0,83 & 4,40 & 1,00 & $t(404)=-39,6 ; p<0,001 ; d=-3,23$ \\
6. Novo emprego & 64,6 & 400 & 4,45 & 0,84 & 1,66 & 0,93 & $t(391)=36,0 ; p<0,001 ; d=3,10$ \\
27. Conflitos com familiares & 58,3 & 361 & 2,13 & 1,52 & 3,88 & 1,10 & $t(358)=-14,4 ; p<0,001 ; d=-1,33$ \\
40. Morte de animal estimação & 57,7 & 357 & 1,37 & 0,73 & 4,27 & 1,06 & $t(352)=-35,2 ; p<0,001 ; d=-3,25$ \\
9. Mudança de status financeiro & 55,6 & 344 & 3,82 & 1,42 & 2,13 & 1,44 & $t(340)=11,5 ; p<0,001 ; d=1,15$ \\
10. Problemas financeiros & 53,5 & 331 & 1,69 & 1,01 & 4,05 & 1,12 & $t(328)=-23,2 ; p<0,001 ; d=-2,21$ \\
17. Foi rejeitado por quem desejava & 53,2 & 329 & 1,85 & 1,15 & 3,92 & 1,13 & $t(326)=-19,9 ; p<0,001 ; d=-1,78$
\end{tabular}


36. Mudança atividades sociais*

18. Reconciliação amorosa

8. Conflitos com chefe

26. Novo membro em casa

53. Ganhou bens

33. Alcoolismo de familiar

25. Demissão de familiar

42. Vítima de assalto

46. Doença física

51. Acidente de carro

3. Mudança de carreira

4. Reprovação em curso

15. Foi infiel*

19. Dificuldades sexuais

49. Abusou álcool, drogas

5. Demissão*

16. Parceiro foi infiel

47. Doença mental

24. Separação, divórcio dos pais

35. Familiar sofreu acidente

30. Família mudou de cidade

13. Casamento

52. Acidente doméstico, trabalho

48. Internação hospitalar

41. Brigou com violência

2. Conclusão curso universitário

21. Gravidez sua ou da parceira

22. Nascimento de filho

20. Separação ou divórcio*

37. Prisão de familiar

43. Vítima de assédio sexual

50. Problemas com uso de drogas, álcool

23. Fez aborto ou sua parceira

34. Filho com problemas emocionais

45. Causou dano ou morte a outra pessoa

38. Violência doméstica, abuso sexual

44. Foi violentado sexualmente
7. Promoção no trabalho

\begin{tabular}{|c|c|c|c|c|c|c|}
\hline 2 & 317 & 3,13 & 1,47 & 2,85 & 1,39 & $t(313)=1,92 ; p=0,056 ; d=0,21$ \\
\hline 0,1 & 310 & 3,82 & 1,21 & 2,20 & 1,22 & $t(305)=13,1 ; p<0,001 ; d=1,31$ \\
\hline 8,6 & 301 & 2,28 & 1,24 & 3,46 & 1,22 & $t(298)=-9,27 ; p<0,001 ; d=-0,9$ \\
\hline 3,9 & 272 & 3,84 & 1,32 & 2,18 & 1,32 & $t(267)=11,0 ; p<0,001 ; d=1,23$ \\
\hline 1 & 242 & 4,74 & 0,62 & 1,34 & 0,78 & $t(238)=46,3 ; p<0,001 ; d=4,90$ \\
\hline 38 & 235 & 1,30 & 0,72 & 4,44 & 0,96 & $t(229)=-33,6 ; p<0,001 ; d=-3,70$ \\
\hline 7,5 & 232 & 1,91 & 1,21 & 3,91 & 1,21 & $t(231)=-14,4 ; p<0,001 ; d=-1,67$ \\
\hline 4,1 & 211 & 1,37 & 0,82 & 4,36 & 1,03 & $t(208)=-29,1 ; p<0,001 ; d=-3,22$ \\
\hline 3,8 & 209 & 1,67 & 0,93 & 3,98 & 1,15 & $t(208)=-18,3 ; p<0,001 ; d=-2,25$ \\
\hline 3,8 & 209 & 1,63 & 1,01 & 3,83 & 1,17 & $t(208)=-18,2 ; p<0,001 ; d=-1,9$ \\
\hline 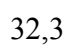 & 200 & 4,06 & 1,16 & 1,82 & 1,12 & $t(195)=16.2 ; p<0,001 ; d=1,92$ \\
\hline 1,3 & 194 & 2,06 & 1,26 & 3,61 & 1,36 & $t(191)=-9,60 ; p<0,001 ; d=-1,1$ \\
\hline 30 & 186 & 2,79 & 1,35 & 3,08 & 1,38 & $t(174)=-1,56 ; p=0,12 ; d=-0,19$ \\
\hline 8,4 & 176 & 4,43 & 0,88 & 1,63 & 1,05 & $t(177)=23,3 ; p<0,001 ; d=2,82$ \\
\hline 8,1 & 174 & 1,64 & 0,98 & 4,06 & 1,11 & $t(172)=-18,1 ; p<0,001 ; d=-2,28$ \\
\hline 7,9 & 173 & 2,74 & 1,22 & 3,23 & 1,26 & $t(171)=-3,06 ; p=0,003 ; d=-0,42$ \\
\hline 5,3 & 163 & 3,12 & 1,46 & 2,84 & 1,48 & $t(160)=1,33 ; p=0,19 ; d=0,20$ \\
\hline 6,2 & 162 & 1,56 & 1,03 & 4,44 & 1,01 & $t(161)=-21,2 ; p<0,001 ; d=-2,7$ \\
\hline 55 & 158 & 1,69 & 0,97 & 4,18 & 1,06 & $t(152)=-16,7 ; p<0,001 ; d=-2,4$ \\
\hline 4,9 & 154 & 2,65 & 1,34 & 3,30 & 1,39 & $t(155)=-3,24 ; p=0,001 ; d=-0$ \\
\hline 3,6 & 146 & 1,37 & 0,90 & 4,39 & 1,09 & $t(144)=-21,6 ; p<0,001 ; d=-2,99$ \\
\hline 3,4 & 145 & 3,68 & 1,32 & 2,45 & 1,28 & $t(140)=6,11 ; p<0,001 ; d=0,97$ \\
\hline 8 & 141 & 4,26 & 1,13 & 2,06 & 1,22 & $t(140)=12,4 ; p<0,001 ; d=1,95$ \\
\hline 5 & 133 & 1,77 & 0,93 & 3,92 & 1,11 & $t(132)=-14,4 ; p<0,001 ; d=-2$ \\
\hline 9,9 & 123 & 2,45 & 1,41 & 3,37 & 1,43 & $t(122)=-3,88 ; p<0,001 ; d=-0,58$ \\
\hline 9,7 & 122 & 2,15 & 1,25 & 3,83 & 1,24 & $t(120)=-8,08 ; p<0,001 ; d=-1,27$ \\
\hline 8,7 & 116 & 4,56 & 0,80 & 1,45 & 0,85 & $t(111)=24,4 ; p<0,001 ; d=3,64$ \\
\hline 7,3 & 107 & 4,46 & 1,19 & 1,67 & 1,23 & $t(103)=12,5 ; p<0,001 ; d=2,21$ \\
\hline . & 95 & 80 & 0,74 & 1,29 & 69 & $t(89)=26,9 ; p<0,001 ; d=4,72$ \\
\hline 13,2 & 82 & 3,05 & 1,57 & 3,04 & 1,59 & $t(79)=0,04 ; p=0,97 ; d=0,15$ \\
\hline 7,6 & 47 & 1,74 & 1,25 & 3,89 & 1,49 & $t(45)=-6,43 ; p<0,001 ; d=-1,62$ \\
\hline 6,9 & 43 & 1,51 & 1,22 & 4,47 & 1,14 & $t(42)=-9,69 ; p<0,001 ; d=-2,41$ \\
\hline 6,6 & 41 & 1,71 & 1,04 & 4,00 & 1,23 & $t(37)=-7,06 ; p<0,001 ; d=-1,97$ \\
\hline 6,1 & 38 & 1,68 & 1,34 & 4,32 & 1,34 & $t(37)=-6,80 ; p<0,001 ; d=-1,86$ \\
\hline 3,9 & 24 & 1,78 & 1,28 & 3,91 & 1,35 & $t(22)=-4,26 ; p<0,001 ; d=-1,54$ \\
\hline 3,2 & 20 & 1,79 & 1,27 & 4,05 & 1,47 & $t(18)=-3,70 ; p=0,002 ; d=-1,30$ \\
\hline 2,4 & 15 & 1,13 & 0,52 & 4,47 & 0,83 & $t(14)=-11,0 ; p<0,001 ; d=-4,54$ \\
\hline 1,8 & 11 & 1,45 & 1,21 & 4,55 & 1,21 & $t(10)=-4,22 ; p=0,002 ; d=-2,36$ \\
\hline
\end{tabular}

Nota. Os itens são apresentados em ordem decrescente, de acordo com o número de participantes que assinalou ter vivenciado. O teste estatístico apresentado refere-se ao teste $t$ pareado, que testa a hipótese nula de igualdade entre as médias da avaliação positiva e negativa.* Os itens $12,36,15,5$ e 20 não mostraram diferenças entre médias. 
Em uma tentativa de realizar uma análise de componentes principais para os 17 eventos vivenciados por mais de $50 \%$ dos participantes, substituíram-se os escores omissos pelas médias das séries, o que para esses 17 itens gerou um número menor de casos hipotéticos. Ainda assim, verificou-se que ao se utilizar as avaliações positivas e negativas dos eventos, as matrizes de correlações mostraram-se inadequadas à fatorização, respectivamente, teste de esfericidade de Bartlett com $p=0,34$ e $p=0,73$; e $\mathrm{KMO}=0,64$ e $\mathrm{KMO}=0,62$. Quando foram utilizados os dados da intensidade dos eventos (multiplicação da avaliação positiva e negativa), constatou-se que o teste de esfericidade de Bartlett mostrou-se significativo, $p=0,002$; porém ainda os dados mostraram-se pouco adequados, $\mathrm{KMO}=0,67$.

Ainda que os dados da intensidade dos eventos fossem ser considerados adequados, os componentes extraídos (seis para eigenvalue $>1$ ) agruparam itens tão diferentes a ponto de considerarem-se inconsistentes as dimensões extraídas. A Tabela 2 apresenta as cargas componenciais dos itens nessas seis dimensões. Por exemplo, os itens 'problemas financeiros', 'término de relação amorosa' e 'conflito com familia- res' carregaram todos em uma mesma dimensão. Ao contrário das classificações em categorias de eventos, frequentemente observadas na literatura, não se verificaram fatores subjacentes capazes de explicar os agrupamentos obtidos, que mais pareceram se comportar de forma aleatória.

Por fim, analisaram-se as respostas a cada evento individualmente. Considerando-se que um mesmo evento foi julgado duas vezes pelas mesmas pessoas, tanto positivamente quanto negativamente, testaram-se as diferenças entre médias das avaliações positivas e negativas para cada um dos eventos através de testes $t$ para dados pareados. Verificou-se uma avaliação positiva superior à negativa para 17 eventos, enquanto para 31 deles a avaliação negativa foi mais elevada. Como pode ser visto também na Tabela 1 , os demais cinco eventos não mostraram diferenças de médias entre as avaliações positivas e negativas, a saber: término de relação amorosa; mudança em atividades sociais; ter sido infiel; ter pedido demissão; ter se separado ou divorciado. Esses achados sinalizam para a possibilidade de alguns eventos serem bidimensionais, uma vez que são avaliados como sendo positivos e negativos ao mesmo tempo.

\section{Tabela 2}

\section{Cargas Componenciais dos Itens de Eventos de Vida}

\begin{tabular}{lcccccc}
\hline & 1 & 2 & 3 & 4 & 5 & 6 \\
\hline 18. Reconciliação amorosa & $\mathbf{0 , 7 5}$ & $-0,20$ & 0,13 & $-0,01$ & 0,05 & 0,12 \\
12. Término de relação amorosa & $\mathbf{0 , 7 2}$ & 0,10 & $-0,01$ & $-0,17$ & $-0,09$ & $-0,03$ \\
14. Conflitos com parceiro & $\mathbf{0 , 5 0}$ & 0,02 & 0,04 & 0,001 & $\mathbf{0 , 3 3}$ & $-0,23$ \\
27. Conflitos com familiares & $\mathbf{0 , 4 8}$ & 0,16 & $\mathbf{- 0 , 4 6}$ & 0,09 & 0,01 & $-0,08$ \\
10. Problemas financeiros & $\mathbf{0 , 3 7}$ & $-0,14$ & $\mathbf{- 0 , 3 4}$ & $\mathbf{0 , 3 7}$ & 0,22 & $\mathbf{- 0 , 3 6}$ \\
11. Início de relação amorosa & $\mathbf{0 , 3 4}$ & 0,09 & 0,29 & 0,28 & $-0,16$ & $-0,13$ \\
32. Familiar doente & 0,13 & $\mathbf{0 , 7 6}$ & $-0,15$ & 0,02 & $-0,23$ & 0,11 \\
40. Morte de animal estimação & $-0,16$ & $\mathbf{0 , 6 9}$ & 0,13 & $-0,06$ & 0,23 & 0,04 \\
39. Falecimento de familiar & $-0,10$ & $\mathbf{0 , 6 1}$ & $-0,02$ & 0,10 & 0,01 & $\mathbf{- 0 , 4 8}$ \\
31. Viagem & 0,06 & $-0,04$ & $\mathbf{0 , 7 4}$ & 0,04 & 0,08 & $-0,07$ \\
29. Mudança de residência & 0,20 & $\mathbf{0 , 3 2}$ & $\mathbf{0 , 4 0}$ & $-0,05$ & 0,12 & $-0,12$
\end{tabular}




\begin{tabular}{|c|c|c|c|c|c|c|}
\hline 9. Mudança de status financeiro & $-0,13$ & 0,03 & $-0,14$ & $\mathbf{0 , 7 7}$ & 0,15 & 0,08 \\
\hline 1. Ingresso curso universitário & $-0,12$ & $-0,07$ & 0,22 & 0,63 & $-0,12$ & $-0,26$ \\
\hline 17. Foi rejeitado por quem desejava & 0,11 & 0,07 & 0,18 & 0,18 & 0,79 & 0,21 \\
\hline 6. Novo emprego & 0,26 & 0,06 & 0,27 & $\mathbf{0 , 3 7}$ & $-0,46$ & 0,23 \\
\hline $\begin{array}{l}\text { 28. Melhoria no relacionamento fa- } \\
\text { miliar }\end{array}$ & 0,14 & 0,36 & $-0,07$ & 0,45 & 0,09 & $\mathbf{0 , 5 8}$ \\
\hline 36. Mudança atividades sociais & 0,15 & 0,11 & 0,12 & 0,14 & $-0,08$ & $-0,57$ \\
\hline
\end{tabular}

Nota. Os escores utilizados nessa análise correspondem à multiplicação da avaliação positiva e negativa sobre cada evento. Cargas maiores que 0,30 estão em negrito.

\section{Discussão}

Um dos objetivos deste estudo foi construir e buscar evidências de validade de uma escala para avaliar a ocorrência e percepção de eventos de vida. No entanto, a partir das análises de componentes principais, não foi possível verificar agrupamentos de itens em dimensões consistentes. Esse resultado sugere que não há evidências de validade de construto para os EV.

Embora inicialmente houvesse a expectativa de obterem-se fatores que agrupassem eventos outrora classificados na literatura em categorias, relativas à educação, trabalho, finanças, saúde, relacionamentos interpessoais etc (ver, por exemplo, Lüdtke et al., 2011; Paykel, 1997), neste estudo, para qualquer conjunto de eventos, a dispersão entre eles demonstrou ser muito grande. Destaca-se que esse procedimento, referente à validade de conteúdo, não garante a consistência e validade de um instrumento psicológico.

Mesmo que na literatura, ao longo dos anos, os EV venham sendo tratados como se constituíssem um construto, os resultados do presente estudo indicam que não há sustentação para tal tratamento sob um ponto de vista psicométrico. O paradoxo não é criado apenas pelo problema metodológico da pesquisa, mas também por um aspecto conceitual: EV constituem ocorrências múltiplas, geradas no ambiente externo ou até mesmo produzidas pelas pessoas. Essas ocorrências são percebidas e vivenciadas subjetivamente e não se configuram como um conceito global.
Em relação à valência atribuída aos eventos, embora os resultados demonstrem uma tendência da amostra consultada em avaliar alguns eventos como mais positivos e outros como mais negativos (17 deles foram avaliados como mais positivos do que negativos, e 31 foram avaliados como mais negativos do que positivos); cinco eventos foram avaliados tanto positivamente quanto negativamente, sem diferenças de médias entre as duas avaliações.

Ou seja, não há como sustentar um padrão de resposta comum para todos os eventos (não se pode assumir que um evento é positivo, negativo ou estressante a priori). Diante da ocorrência de um evento, é necessário considerar o valor atribuído pelo respondente. Somente este poderá nos dizer se o término de uma relação amorosa, por exemplo, foi um evento positivo ou negativo em sua vida, bem como, o quão positiva e negativa foi esta vivência.

Desta forma, os resultados do presente estudo reforçam a necessidade de considerar-se na pesquisa em EV, a percepção e avaliação subjetiva dos eventos. Sendo assim, é necessário cautela ao se classificarem EV de acordo com uma suposta valência hedônica ou desejabilidade compartilhada; distinguindo os eventos em positivos, negativos e neutros (ver Filipp \& Aymanns, 2009; Luhmann et al., 2012).

\section{Conclusão}

Por meio dos resultados obtidos neste estudo, temos uma evidência empírica demonstran- 
do que não há agrupamento consistente e com sentido teórico para os eventos vividos na população estudada. Cada evento de vida é também construído por cada um, constituindo uma experiência subjetiva, embora possa ser ativado no ambiente externo. Então, deparamo-nos com a dificuldade de construção de uma escala que os agrupe em fatores.

Sendo assim, questiona-se a condução de alguns estudos epidemiológicos com base em escalas de eventos. Em geral, as pesquisas envolvendo EV não informam sobre as propriedades psicométricas dos instrumentos utilizados. Em muitos casos, os EV são tratados como se fossem construtos na medida em que são agrupados em dimensões sem referência à validade de construto e conteúdo. Em outros casos, escores totais positivos e negativos são derivados das listas, desconsiderando-se os eventos específicos e as avaliações subjetivas dos respondentes quanto à percepção dos eventos.

Além disso, não foi identificada na literatura nenhuma escala que contemplasse a avaliação positiva e negativa dos eventos simultaneamente. Os resultados do presente estudo demonstram que nenhum evento é apenas negativo ou positivo. Em geral, os eventos foram avaliados como sendo ou mais positivos ou mais negativos, variando na intensidade da avaliação positiva e negativa. Contudo, para cinco deles não houve diferença significativa nas médias das avaliações positivas e negativas. Diante disso, considera-se importante insistir na individualidade do dado e da informação, reconhecendo na avaliação de EV a interação entre o sujeito e os eventos, investigando-se as percepções individuais e capacidades de resposta.

Como limitações do presente estudo, podemos citar a constituição da amostra por conveniência e a sua ampla faixa etária, o fato de não se ter avaliado quando os eventos ocorreram e de não se ter quantificado o número de ocorrências dos eventos. Sugerimos que em pesquisas futuras, essas variáveis sejam estudadas. Além disso, sugere-se que, ao se estudar a relação dos EV com outras variáveis, tais como BES, Personalidade, Coping e o advento de afecções psíquicas, seja melhor investigado o impacto das contribui- ções relativas à ocorrência dos EV e à avaliação subjetiva dos EV em relação ao BES.

Por exemplo, o que impacta mais no BES, a ocorrência de determinado evento ou a forma como ele é percebido e valorizado por um sujeito? Além disso, em que medida EV são independentes de traços de personalidade? Sugerimos, em estudos futuros, a investigação da relação entre esses fenômenos, bem como, um aprofundamento sobre as implicações da inclusão de avaliações positivas e negativas, separadamente, para um mesmo evento.

\section{Referências}

Bifulco, A., Brown, G., Edwards, A., Harris, T., Neilson, E., Richards, C., \& Robinson, R. (1989). Life events and difficulties schedule (LEDS-2): Vol. 1. Life events manual. London: Bedford New College, University of London.

Brown, G. W., \& Harris, T. (1978). Social origins of depression. London: Tavistock.

Chalise, H. N., Saito, T., Takahashi, M., \& Kai, I. (2007). Relationship specialization amongst sources and receivers of social support and its correlations with loneliness and subjective wellbeing: A cross sectional study of Nepalese older adults. Archives of Gerontology and Geriatrics, 44(3), 299-314.

Dell'Aglio, D. D., Benetti, S. P., Deretti, L., D’Incao, D. B., \& Leon, J. S. (2005). Eventos estressores no desenvolvimento de meninas adolescentes cumprindo medidas sócio-educativas. Paidéia (Ribeirão Preto), 15(30), 119-129.

Diener, E., \& Oishi, S. (2005). The nonobvious social psychology of happiness. Psychological Inquiry, 16(4), 162-167.

Dohrenwend, B. S. (1985). Social status and responsibility for stressful life events. Issues of Mental Health and Nurse, 7, 105-127.

Dohrenwend, B. S. (1998). Adversity, stress, and Psychopathology. New York: Oxford University Press.

Dohrenwend, B. P. (2006). Inventorying stressful life events as risk factors for psychopathology: Toward resolution of the problem of intracategory variability. Psychological Bulletin, 132(3), 477-495. 
Faravelli, C., Catena, M., Scarpato, A., \& Ricca, V. (2007). Epidemiology of life events: Life events and psychiatric disorders in the Sesto Fiorentino Study. Psychotherapy and Psychosomatics, 76(6), 361-368.

Farmer, A., Redman, K., Harris, T., Mahmood, A., Sadler, S., Pickering, A., \& McGuffin, P. (2002). Neuroticism, extraversion, life events and depression. The British Journal of Psychiatry, 181, $118-122$

Filipp, S., \& Aymanns, P. (2009). Critical life events and life crises: Coping with the dark sides of life. Stuttgart, Germany: Kohlhammer.

Fortes-Burgos, A. C. G., Neri, A. L., \& Cupertino, A. P. F. B. (2009). Eventos de vida estressantes entre idosos brasileiros residentes na comunidade. Estudos de Psicologia (Natal), 14(1), 69-75.

Fountoulakis, K. N., Iacovides, A., Kaprinis, S., \& Kaprinis, G. (2006). Life events and clinical subtypes of major depression: A cross-sectional study. Psychiatry Research, 143, 235-244.

Garcia, C. R. (2007). Eventos de vida em pacientes adultos com transtorno de déficit de atenção/ hiperatividade (Dissertação de mestrado, Programa de Pós-Graduação em Ciências Médicas: Psiquiatria, Universidade Federal do Rio Grande do Sul, Porto Alegre, RS, Brasil).

Gomez, V., Krings, F., Bangerter, A., \& Grob, A. (2009). The influence of personality and life events on subjective well-being from a life span perspective. Journal of Research in Personality, 43, 345-354.

Headey, D., \& Wearing, A. (1989). Personality, life events, and subjective well-being: Toward a dynamic equilibrium model. Journal of Personality and Social Psychology, 57(4), 731-739.

Holmes, T. H., \& Rahe, R. H. (1967). The social readjustment rating scale. Journal of Psychosomatic Research, 11, 213-218.

Jordanova, V., Stewart, R., Goldberg, D., Bebbington, P. E., Brugha, T., Singleton, N., ...Meltzer, H. (2007). Age variation in life events and their relationship with common mental disorders in a national survey population. Social Psychiatry and Psychiatric and Epidemiology Journal, 611-616.

Kessler, R. C. (1997). The effects of stressful life events on depression. Annual Review of Psychology, 48, 191-214.
Kohn, Y., Zislin, J., Agid, O., Hanin, B., Troudart, T., Shapira, B., ...Lerer, B. (2001). Increased prevalence of negative life events in subtypes of major depressive disorder. Comprehensive Psychiatry, 42(1), 57-63.

Kolarik, B. (2010). The relation of recalled life events, childhood relationships with parents and meaning in life. Research on Steiner Education, 1(2), 96-116.

Leskela, U. S., Melartin, T. K., Lestela-Mielonen, P. S., Rytsala, H. J., Sokero, T. P., Heikkinen, M. E., \& Isometsa, E. T. (2004). Life events, social support, and onset of major depressive episode in Finnish patients. Journal of Nervous Mental Disorders, 192(5), 373-381.

Lüdtke, O., Roberts, B. W., Trautwein, U., \& Nagy, G. (2011). A random walk down university avenue: Life paths, life events, and personality trait change at the transition to university life. Journal of Personality and Social Psychology, 101(3), 620-637.

Luhmann, M., Hofmann, W., Eid, M., \& Lucas, R. E. (2012). Subjective well-being and adaptation to life events: A meta-analysis. Journal of Personality and Social Psychology, 102(3), 592-615.

Margis, R., Picon, P., Cosner, A. F., \& Silveira, R. O. (2003). Stressfull life-events, stress and anxiety. Revista de psiquiatria Rio Grande do Sul, 25(1), 65-74.

Morse, J. Q., \& Robins, C. J. (2005). Personality-life event congruence effects in late-life depression. Journal of Affective Disorders, 84, 25-31.

Murray, W. E., \& Cox, B. J. (2005). Psychosocial and clinical predictors of symptom persistence vs remission in major depressive disorder. The Canadian Journal of Psychiatry, 50, 769-777.

Oliveira, M. G., Fonseca, P. P., \& Del Porto, J. A. (2002). Versão brasileira da entrevista de Paykel para eventos de vida recentes. Revista Brasileira de Terapia Comportamental e Cognitiva, 4(1), 47-61.

Paykel, E. S. (1994). Life events, social support and depression. Acta Psychiatrica Scandinavica Supplement, 377, 50-58.

Paykel, E. S. (1997). The interview for recent life events. Psychological Medicine, 27(2), 301-310.

Paykel, E. S. (2003). Life events and affective disorders. Acta Psychiatrica Scandinavica Supplement, 418, 61-66. 
Paykel, E. S., Rao, B. M., \& Taylor, C. N. (1984). Life stress and symptom pattern in outpatient depression. Psychological Medicine, 14, 559568.

Rafanelli, C., Roncuzzi, R., Milaneschi, Y., Tomba, E., Colistro, M. C., Pancaldi, L. G. \& Di Pasquale, G. (2005). Stressful life events, depression and demoralisation as risk factors for acute coronary heart disease. Psychotherapy and Psychosomatics, 74(3), 179-184.

Reich, J. W., \& Zautra, A. (1981). Life events and personal causation: Some relationships with satisfaction and distress. Journal of Personality and Social Psychology, 41(5), 1002-1012.

Sarason, I. G., Johnson, J. H., \& Siegel, J. M. (1978). Assessing the impact of life changes: Development of the life experiences survey. Journal of Consulting and Clinical Psychology, 46, 932946.

Savoia, M. G., \& Bernik, M. (2004). Adverse life events and coping skills in panic disorder. Revista do Hospital de Clínicas da Faculdade de Medicina de São Paulo 59(6), 337-340.

Schimmack, U., \& Diener, E. (2003). Predictive validity of explicit and implicit self-esteem for subjective well being. Journal of Research in Personality, 37(2), 100-106.
Sheril, J., Cueller, A. K., Ruggero, C., Winett-Perlman, C., Goodnick, P., White, R., \& Miller, I. (2008). Life events as predictors of mania and depression in bipolar i disorder. Journal of $\mathrm{Ab}$ normal Psychology, 117(2), 268-277.

Spurgeon, A., Jackson, C. A., \& Beach, J. R. (2001). The life events inventory: Re-scaling based on an occupational sample. Occupational Medicine, 51(4), 287-293.

Tessner, K. D., Mittal, V., \& Walker, E. F. (2011). Longitudinal Study of Stressful Life Events and Daily stressors among Adolescents at High Risk for Psychotic Disorders. Schizophrenia Bulletin, 31(2), 432-441.

Voss, E., Stegmann, A., \& Schroder, J. (2006). Stressful life events, protective factors and depressive disorders in middle adulthood. In H. W. Wahl, H. Brenner, H. Mollenkopf, D. Rothenbacher, \& C. Rott (Eds.), The many faces of health, competence and well-being in old age: Integrating epidemiological, psychological and sociological perspectives (pp. 99-113). Dordrecht, Netherlands: Springer. 